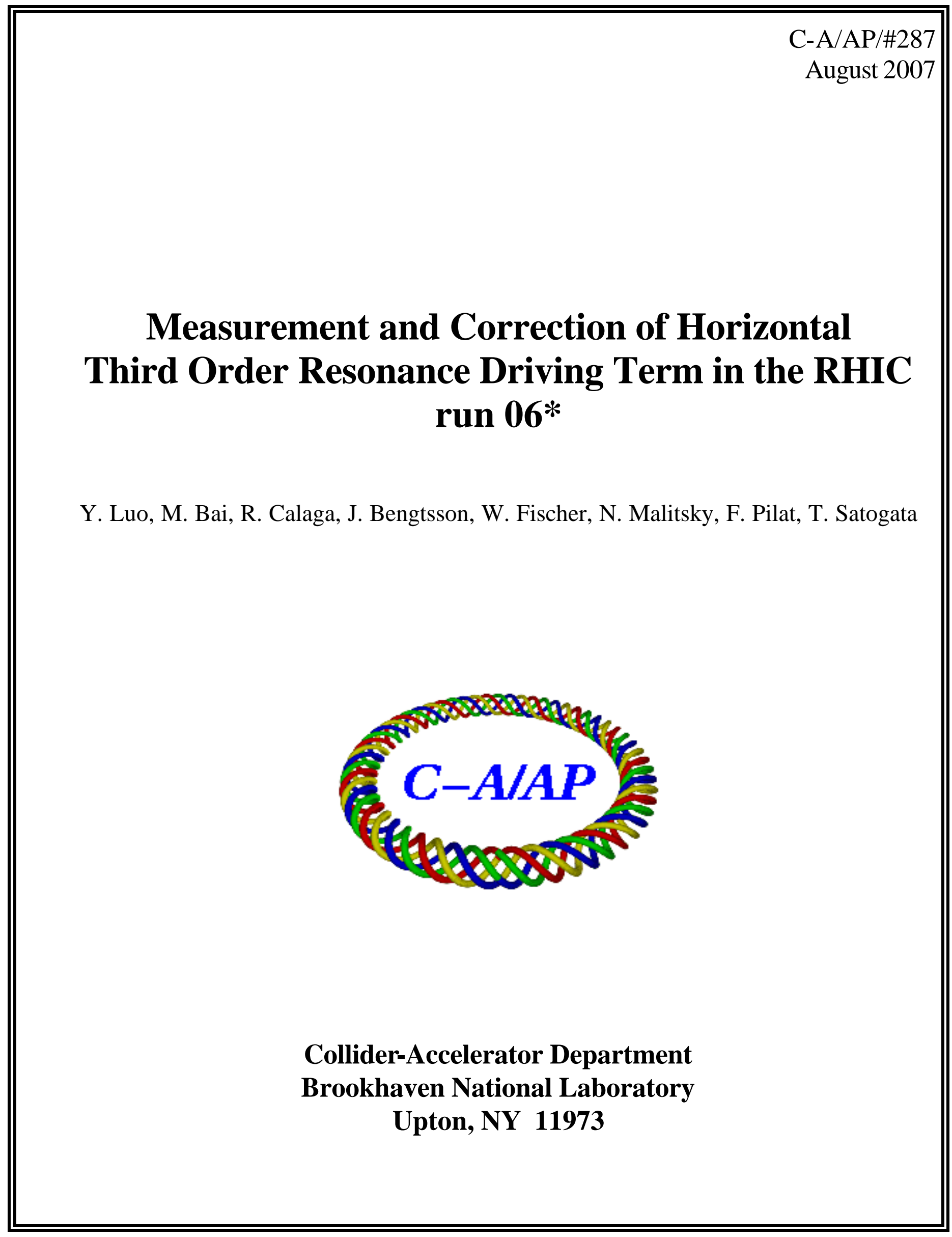




\title{
Measurement and Correction of Horizontal Third Order Resonance Driving Term in the RHIC run 06*
}

\author{
Y. Luo, M. Bai, R. Calaga, J. Bengtsson, W. Fischer, N. Malitsky, F. Pilat, T. Satogata \\ Brookhaven National Laboratory, Upton, NY 11973, USA
}

\section{Abstract}

To further improve the luminosity in polarized proton operation of the Relativistic Heavy Ion Collider, correction of the horizontal two-third resonance at store is desirable. The long-lasting coherent beam oscillations, produced by the AC dipole, are used to measure $3 Q_{x}$ 's resonance driving term $h_{30000}$ through the analysis of turn-by-turn beam position data with the algorithm outlined by J. Bengtsson. The resonance driving term $h_{30000}$ can be compensated with the 12 arc chromatic sextupole families. In this article, we will review the technique of $h_{30000}$ resonance driving term's measurement and correction with AC dipole excitation in the RHIC, followed by the preliminary beam experiment results from the RHIC 2006 polarized proton run.

\section{INTRODUCTION}

To increase the tune space available for beam-beam generated tune spread, a third order resonance correction at the current working point $\left(Q_{x}=28.685, Q_{y}=29.695\right)$ for polarized proton (pp) operation in the Relativistic Heavy Ion Collider (RHIC) is desirable [1]. At the current working point the fractional horizontal tune $Q_{x}$ is constrained by the third order resonance $Q_{x}=2 / 3$, and the tenth order resonance $Q_{x}=0.7$. With $Q_{x}$ close to 0.7 , both luminosity and polarization suffer. A further increase in the bunch intensity will push the particles in the center of the bunch to third order resonance $Q_{x}=2 / 3$.

In Ref. [2], the basic theory of the sextupole first order driving terms is outlined, and an algorithm is provided to extract first order driving terms from turn-by-turn (TBT) actions $J_{x}(N)$. With a modified Fast Fourier Transformation (FFT) technique, the betatron tune $Q_{x}$ in the spectrum of $x$, and the $3 Q_{x}$ peak in the spectrum of the action $J_{x}(N)$, can be precisely calculated [3]. According to Ref. [2], the peak at $3 Q_{x}$ in the spectrum of $J_{x}(N)$ is linked to the $3 Q_{x}$ resonance.

To be able to extract $h_{30000}$ from TBT beam position monitor (BPM) data, a long-lasting, coherent, and large amplitude betatron oscillation is required. At $100 \mathrm{GeV}$, after 5 consecutive kicks with the tune meter kicker, the maximum oscillation amplitude of the beam center is about $1.5 \mathrm{~mm}$ at BPM rbpm.bo6-bh4. A disadvantage of the kicked BPM data is its fast decoherence and small amplitude. In addition, too many kicks increase the emittance significantly.

\footnotetext{
* This work was supported by Brookhaven Science Associates, LLC under Contract No. DE-AC02-98CH10886 with the U.S. Department of Energy and by a sponsored research grant from Renaissance Technologies Corporation.
}

The AC dipole has been proven to be a powerful tool to produce long-lasting coherent beam oscillations with large amplitude. If the driving strength of $\mathrm{AC}$ dipole is ramped up and down adiabatically, no significant emittance increase is observed [4]. The TBT BPM data taken at the flattop of AC dipole excitation can be used for linear as well as nonlinear lattice measurements $[5,6]$.

In the 2006 polarized proton run, dedicated beam experiments were carried out at the RHIC injection to verify the technique to measure the thrid order resonance driving term $h_{30000}$ with the TBT BPM data produced the AC dipole excitation. The 12 chromatic sextupole families were used to correct the $3 Q_{x}$ driving term $h_{30000}$ at the observation point, while keeping the first order chromaticities and other 5 first order sectupole driving terms unchanged. In this optimization there are totally 12 variables and 12 constraints.

In the following, we first review the principle of measurement and correction of the third order resonance driving term $h_{30000}$. Then, the preliminary results from the beam experiments in the RHIC 2006 pp run were presented and the challenge in this technique is discussed.

\section{BACKGROUNDS}

\section{First order resonance driving terms}

Sextupoles produce the following first order geometric driving terms,

$$
\begin{aligned}
h_{21000} & =-\frac{1}{8} \sum_{i=1}^{N}\left(k_{2} d l\right)_{i} \beta_{x, i}^{3 / 2} e^{i \mu_{x, i}}, \\
h_{30000} & =-\frac{1}{24} \sum_{i=1}^{N}\left(k_{2} d l\right)_{i} \beta_{x, i}^{3 / 2} e^{i 3 \mu_{x, i}}, \\
h_{10110} & =\frac{1}{4} \sum_{i=1}^{N}\left(k_{2} d l\right)_{i} \beta_{x, i}^{1 / 2} \beta_{y, i} e^{i \mu_{x, i}}, \\
h_{10020} & =\frac{1}{8} \sum_{i=1}^{N}\left(k_{2} d l\right)_{i} \beta_{x, i}^{1 / 2} \beta_{y, i} e^{i\left(\mu_{x, i}-2 \mu_{y, i}\right)}, \\
h_{10200} & =\frac{1}{8} \sum_{i=1}^{N}\left(k_{2} d l\right)_{i} \beta_{x, i}^{1 / 2} \beta_{y, i} e^{i\left(\mu_{x, i}+2 \mu_{y, i}\right)} .
\end{aligned}
$$

They will drive the resonances $Q_{x}, 3 Q_{x}, Q_{x}, Q_{x}-2 Q_{y}$, and $Q_{x}+2 Q_{y}$, respectively, and are therefore called resonance driving terms. From Eqs. (1)-(5), each resonance driving term is a complex number,

$$
h_{i j k l 0}=A_{i j k l 0} e^{i \phi_{i j k l 0}} .
$$

$A_{i j k l 0}$ and $\phi_{i j k l 0}$ are the amplitude and phase of the driving term $h_{i j k l 0}$, respectively. 
According to Refs. [2], the perturbed betatron motion with first order resonance driving terms are:

$$
\begin{aligned}
& J_{x}(N)=J_{x} \\
& +\frac{3 A_{30000}\left(2 J_{x}\right)^{3 / 2}}{\sin \left(3 \pi Q_{x}\right)} \cos \left[\hat{\phi}_{30000}+3 \phi_{x}+2 \pi N \cdot 3 Q_{x}\right] . \\
& +\ldots
\end{aligned}
$$

where $J_{x}(N)$ is the horizontal and vertical actions of the $N$ th turn. The $\phi_{x}$ and $\phi_{y}$ are phases of the horizontal and vertical betatron oscillations. In Eqs. (7), $J_{x}$ is the average actions.

\section{$A C$ dipole excitation}

In beam experiment, the AC dipole is used here to produce the long-lasting coherent beam oscillation BPM data from which $h_{30000}$ can be extracted. By exciting the beam with an $\mathrm{AC}$ dipole at a frequency in the vicinity of the betatron frequency, a coherent beam oscillation with large amplitude can be generated at the drive frequency. If the amplitude of the AC dipole strength is ramped up and down adiabatically, there is no significant emittance increase. In RHIC, the AC dipole drive tune is typically $Q_{d}=Q x \pm 0.01$. The AC dipole strength ramps from zero to its maximum value in about 6000 turns, or $76 \mathrm{~ms}$. The TBT BPM data are taken at the flattop of the AC dipole excitation.

\section{Extracting $h_{30000}$}

On either side of the RHIC IRs, there are two dual-plane BPMs, located between quadrupoles Q3 and Q4. These two BPMs, rbpm.bo6-bh3 and rbpm.bo6-bh4, are separated by a $36.2984 \mathrm{~m}$ long drift. A construction of the $\left(x, p_{x}\right)$ and $J_{x}(N)$ from the 1024 turn-by-turn data at the horizontal ' $x$-position readings' of the BPM rbpm.bo6-bh3 was done in the $h_{30000}$ measurement. The horizontal Twiss parameters can be derived with an ellipse fitting, or a harmonic analysis with the 1024 TBT BPM data.

According to Eq. (7), we can determine the amplitude and phase of the $3 Q_{x}$ resonance driving term $h_{30000}$ with an FFT of the TBT action $J_{x}(N)$. The amplitude and phase of the $3 Q_{x}$ peak in the $J_{x}(N)$ spectrum are $A_{3 Q_{x}}$ and $\Phi_{3 Q_{x}}$, respectively. Then, the amplitude and the phase of $h_{30000}$ are given by

$$
\begin{gathered}
A_{30000}=\kappa * \sin \left(3 \pi Q_{x}\right) / 3, \\
\phi_{30000}=\Phi_{3 Q_{x}}-3 \phi_{x},
\end{gathered}
$$

where $\kappa$ is the amplitude ratio of the $Q_{x}$ and $3 Q_{x}$ peaks in the spectrum of $J_{x}(N)$. Note that the $Q_{x}$ peak in the spectrum of $J_{x}(N)$ is located at zero frequency, which gives the average of $J_{x}(N)$, or $J_{x}$ in In Eqs. (7) . For Eq. (10), we have assumed that $3 Q_{x} \approx p$, with $p$ being an integer.

\section{Correcting $h_{30000}$}

There are a total of 144 sextupole magnets in the 6 arcs of each RHIC ring. In the previous runs, only two fami- lies, one focusing and one defocussing, were used for the first order chromaticity correction. In an attempt to correct the third order resonance driving term $h_{30000}$ in Run 2006, we split the arc sextupoles into 12 sub-families since there are a total of 12 arc sextupole power supplies in each ring. This correction scheme allowed us to control the two first order chromaticities, and the other five complex first order resonance driving terms $(5 * 2)$ from sextupoles. The singular value decomposition (SVD) technique is used for this optimization.

Simulations of the third resonance driving term measurement with AC dipole and corrections with different schemes were given in Ref. [7, 8].

\section{BEAM EXPERIMENT RESULTS}

In the RHIC 2006 run, dedicated beam experiments were carried out at the injection to verify the method to measure and correct the third order resonance $3 Q_{x}$ 's driving term $h_{30000}$ with the AC dipole excitation. The key point to this etchnique is to obtain the useful TBT BPM data and extract solid $h_{30000}$ resonance driving term, including its amplitude and phase. Based on the measurement of $h_{30000}$, its correction can be applied with the 12 arc chromatic sextupole families.

In the beginning of this beam experiments, the TBT BPM data from the kicked beam by the RHIC tune meter kicker were used to extract $h_{30000}$ resonance driving term. At the injection, after 5 kickings in 5 consecutive turns, the maximum oscillation amplitude of the kicker bunch center can reach $1.2 \mathrm{~mm}$, which was not sufficient to extract the $h_{30000}$ resonance driving term. And the decoherence after kickings also prevented us using these TBT BPM data from kicked beam oscillation.

Then, we used the RHIC AC dipole to coherently excite the beam to produce high quality long-lasting coherent TBT BPM data. The AC dipole excitation tune was chosen to be \pm 0.1 away from the betatron tune. In the beam experiments, intolerable beam time deterioration was observed when the beam was excited to higher oscillation amplitudes. This may be due to the poor beam dynamic apertures in the RHIC 2006 pp run. The maximum oscillation amplitude of the bunch center in the triplets in our beam experiments was below $3.0 \mathrm{~mm}$. During the TBT BPM data were taken, only horizontal beam oscillation was excited with AC dipole. The vertical tune was set far away from the vertical third order resonance $3 Q_{y}$.

The AC dipole excited TBT BPM data at rbpm.bo6-bh3 and rbpm.bo6-bh4 were taken while we were pushing the beam to the $3 Q_{x}$ resonance line. At each horizontal tune, the AC dipole was fired with different excitation strengths. And we also measured $h_{30000}$ resonance driving term when we included extra cosine and sine terms of $h_{30000}$, using the 12 arc sextupole families with the $h_{30000}$ response matrix.

As an example, Fig. 1 shows the TBT normalized coordinates $\left(x_{n}, x_{p n}\right)$ at BPM rbpm.bo6-bh3 for data set “acd_H_2nux+0.01_0.316_inj_29.sdds". Fig. 2 shows the 
TBT action $J_{x}(N)$ for same data set. This data set were taken under the condition that the horizontal betatron tune was .674 and the AC dipole driving tune was 0.684 . Fig. 3 shows the spectrum of $J_{x}(N)$. In Fig. 3, the $3 Q_{x}$ peak at tune $3 \times 0.684-2=0.052$ can be clearly seen.

For most of the data sets we took, the $3 Q_{x}$ peaks did show up in the spectrum of $J_{x}(N)$. However, we found that the measured amplitudes and phases of $h_{3000}$ didn't repeat very well from measurement to measurement, although more measurements at same beam and AC dipole extitation conditions helped some. The unsatisfactory S/N in the measurement of $h_{30000}$ may be linked to the the small oscillation amplitudes of the bunch center. The third order resonance motion only shows up with large beam oscillation amplitude. And during these beam experiments, malfunction of the low level signal generator in the $\mathrm{AC}$ dipole system was reported. Therefore, the technique of measuring $h_{30000}$ driving term with $\mathrm{AC}$ dipole excitation was not well established in the RHIC run 06. This also prevented applying affective $h_{30000}$ correction in these beam experiements.

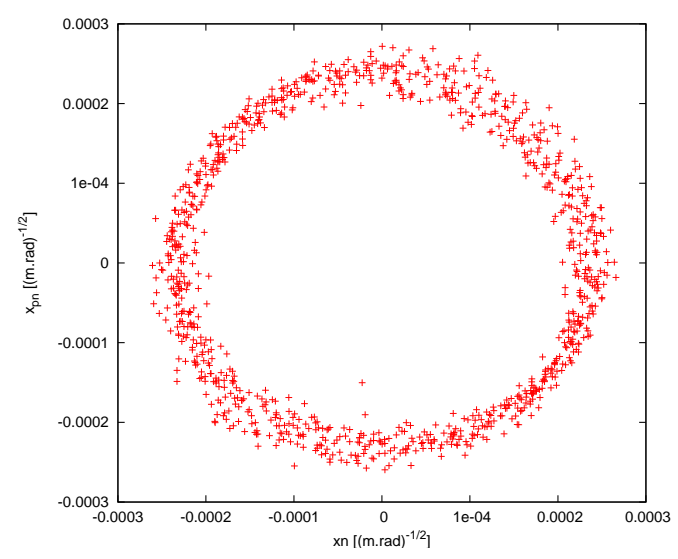

Figure 1: The TBT normalized coordinates $\left(x_{n}, x_{p n}\right)$.

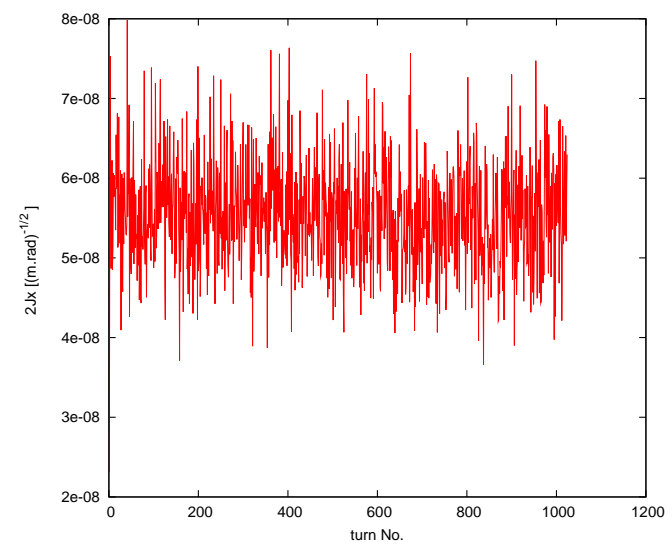

Figure 2: The TBT action $J_{x}(N)$.

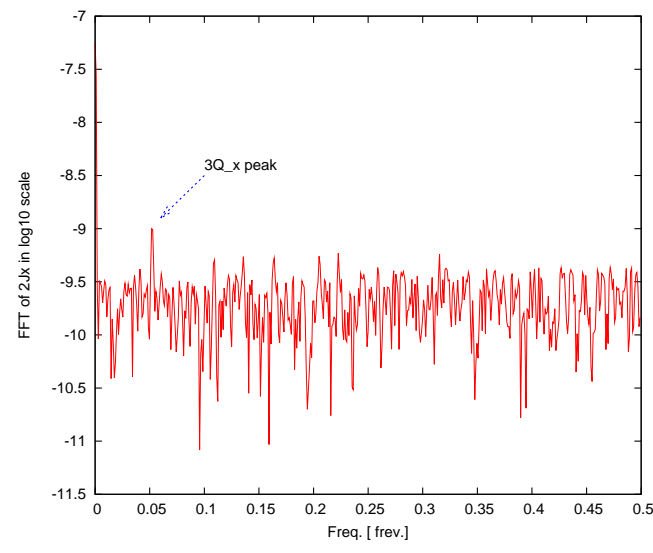

Figure 3: The spectrum of $J_{x}(N)$.

\section{CONCLUSION}

To further improve the luminosity in polarized proton operation of the Relativistic Heavy Ion Collider, correction of the horizontal two-third resonance at the current working point in store is desirable. Dedicated beam experiments were carried out at the injection in the RHIC 2006 pp run to verify the method to measure and correct the third order resonance $3 Q_{x}$ 's driving term $h_{30000}$ suggested by $\mathbf{J}$. Bengtsson in Ref. [2]. The Ac dipole was used to produce the long-lasting coherent TBT BPM data. $h_{30000}$ can be extracted from the spectrum of TBT action $J_{x}(N)$. The $3 Q_{x}$ peak in the spectrum of TBT action $J_{x}(N)$ was observed in the beam experiments. However, the unsatisfactory $\mathrm{S} / \mathrm{N}$ signal in the $h_{30000}$ measurement prevented affective $h_{30000}$ correction in these beam experiments.

\section{REFERENCES}

[1] W. Fischer, Beam-beam and BTF, 2006 RHIC Accelerator Physics Experiments Workshop, November 2-3, 2005, BNL.

[2] J. Bengtsson, The sextupole scheme for the Swiss Light Source(SLS): an analytical approach, SLS Note 9/97, March 7, 1997.

[3] J. Laskar, Physica D 67, 257 (1993).

[4] M. Bai, et al., Experimental test of coherent betatron resonance excitation, Phys. Rev. Lett. 56, 6002, 1999.

[5] F. Schmidt, R. Tomas, Measurement of drving terms, Proceedings of the 2001 Particle Accelerator Conference, Chicago, 2001.

[6] R. Tomas, et al., Measurement of global and local resonance terms, Phys. Rev. ST Accel. Beams, 8, 024001(2005).

[7] Y. Luo, M. Bai, J. Bengtsson, W. Fischer, D. Trbojevic, Simulation of $3 Q_{x}$ resonance driving term measurement, BNL C-AD AP Note-265, Dec., 2006.

[8] Y. Luo, J. Bentsson, W. Fischer, and D. Trbojevic, Simulation of proposed on-line third order resonance correction schemes, BNL C-AD AP Note-264, Dec., 2006. 